\title{
Social anxiety disorder: radio electric asymmetric conveyor brain stimulation versus sertraline
}

This article was published in the following Dove Press journal:

Patient Preference and Adherence

2I November 201।

Number of times this article has been viewed

\author{
Vania Fontani' \\ Piero Mannu',2 \\ Alessandro Castagna' \\ Salvatore Rinaldi' \\ 'Department of Neuro Psycho Physio \\ Pathology, Rinaldi Fontani Institute, \\ Florence; ${ }^{2}$ Psychic Studies Center, \\ Cagliari, Italy
}

Purpose: Social anxiety disorder (SAD) is a disabling condition that affects almost $5 \%$ of the general population. Many types of drugs have shown their efficacy in the treatment of SAD. There are also some data regarding psychotherapies, but no data are available today about the efficacy of brain stimulation techniques. The aim of the study is to compare the efficacy of noninvasive brain stimulation neuro psycho physical optimization (NPPO) protocol performed by radio electric asymmetric conveyor (REAC) with that of sertraline in adults with SAD.

Patients and methods: Twenty SAD patients on sertraline were compared with 23 SAD patients who refused any drug treatment and who chose to be treated with NPPO-REAC brain stimulation. This was a 6-month, open-label, naturalistic study. Patients on sertraline received flexible doses, whereas NPPO-REAC patients received two 18-session cycles of treatment. Clinical Global Improvement scale items "much improved" or "very much improved" and Liebowitz Social Anxiety Scale total score variation on fear and avoidance components were used to detect the results. The statistical analysis was performed with $t$-test. All measures $<0.05$ have been considered statistically significant.

Results: Ten of 23 subjects on NPPO-REAC and six of the 20 taking sertraline were much improved or very much improved 1 month after the first NPPO-REAC cycle (t1). Sixteen of the subjects on NPPO-REAC and ten of the subjects taking sertraline were much improved or very much improved 1 month after the second NPPO-REAC cycle (t2). In respect of the Liebowitz Social Anxiety Scale, at t1 NPPO-REAC resulted in statistically more efficacy for sertraline on both fear and avoidance total scores. At 2 , NPPO-REAC resulted in statistically more efficacy for sertraline on fear but not on avoidance.

Conclusion: NPPO-REAC is an effective treatment for SAD, allowing substantial and clinically meaningful reductions in symptoms and disability in comparison with sertraline.

Keywords: social anxiety disorder, brain stimulation, REAC, sertraline, fear, avoidance

\section{Introduction}

Social anxiety disorder (SAD), also known as social phobia, is characterized by the marked fear of being observed or evaluated by others, ${ }^{1-3}$ in particular nonrelatives. In such situations, patients with SAD fear that they will say or do something to embarrass or humiliate themselves or that others will notice that they are anxious. Consequently, subjects with SAD often avoid situations ${ }^{2,4,5}$ where such scrutiny might take place, or they endure them with intense distress. ${ }^{6}$ This can result in impaired functioning and disrupted quality of life. ${ }^{7,8}$ Patients affected from SAD may have few social relationships, experience trouble dating, drop out of school ${ }^{9,10}$ or work, ${ }^{11}$ reject promotions at work, become demoralized, abuse alcohol, ${ }^{12-16}$ and develop other psychiatric disorders ${ }^{17-19}$ like major depression. ${ }^{20-22} \mathrm{SAD}$ is more frequent in
Correspondence: Salvatore Rinald Rinaldi Fontani Institute, Viale Belfiore 43, 50144 Florence, Italy

Tel +39055 290307

Fax +39055 290399

Email srinaldi@irf.it 
the primary care setting, ${ }^{23-27}$ but it is often undiagnosed ${ }^{28}$ and, consequently, untreated. This low rate of recognition and appropriate treatment reflects the fact that social phobia remains a largely neglected anxiety disorder. ${ }^{29.30}$ However, the available epidemiological studies ${ }^{31-33}$ show a prevalence of at least $5 \%$ in the general population.

Most clinicians associate the term "social phobia" with a fear of public speaking. Indeed, social phobia often involves public speaking and, in some cases, does so exclusively. However, there is a variant of SAD that is more pervasive and usually more disabling: generalized $\mathrm{SAD}^{7,34-37}$ (gSAD). Subjects with gSAD typically fear and avoid a broad array of situations that most people take for granted, such as speaking in small groups, attending social gatherings, talking to people in authority, and interacting with peers in an informal setting.

The neglect of gSAD obviously extends into the area of treatment. Treatment options for gSAD include monoamine oxidase inhibitors, ${ }^{38}$ reversible inhibitors of monoamine oxidase A (moclobemide), ${ }^{39,40}$ and, in particular, selective serotonin reuptake inhibitors. ${ }^{41-43}$ Based on their success in the treatment of many mood and other anxiety disorders, selective serotonin reuptake inhibitors have been investigated in the treatment of social phobia, and they are considered as first-choice drugs for the treatment of gSAD. Excluding some psychotherapies, ${ }^{4-47}$ at the moment, no data are available about other therapeutical approaches such as noninvasive brain stimulation techniques like radio electric asymmetric conveyor (REAC) treatments. Neuro psycho physical optimization (NPPO)-REAC has demonstrated efficacy in improving certain psychiatric disorders such as stress-related disorders, ${ }^{48-53}$ anxiety, ${ }^{53,54}$ depression, ${ }^{53-55}$ bipolar disorder, ${ }^{56}$ and behavioral and psychiatric symptoms in Alzheimer disease ${ }^{57}$ The main goal of the present study was to investigate the efficacy of NPPO-REAC in the treatment of gSAD in patients who refuse drug treatment.

\section{Materials and methods}

This was an open-label, naturalistic study. Patients with gSAD came spontaneously to our medical centers and were observed in the normal clinical practice. gSAD was diagnosed with structured clinical interview ${ }^{58,59}$ according to the Diagnostic and Statistical Manual of Mental Disorders, Fourth Edition, Text Revised.

The data for the current study were collected from the Psychic Studies Center, Cagliari, Italy, for 20 patients (Table 1) treated with flexible doses of sertraline ${ }^{60-62}$ (mean dose $125.0 \pm 15.5 \mathrm{mg} /$ day once a day) and from the Rinaldi
Fontani Institute, Florence, Italy, for 23 patients (Table 1) who refused drug treatment and were treated with two cycles of 18 NPPO-REAC sessions. The time between the two treatment cycles was about 3 months. None of the patients enrolled in the study had been previously treated for gSAD, and none took psychotropic drugs during the study, except for sertraline. Patients were evaluated for safety and efficacy about 1 month after the end of the first NPPO-REAC treatment cycle ( $\mathrm{t} 1$ ) and about 1 month after the end of the second NPPO-REAC treatment cycle (t2). According to the RinaldiFontani protocol, this time period lasted about 6 months and determined the duration of the comparison study. The main efficacy variables were the percentage of responders at $t 1$ and t2, defined as those rated on the Clinical Global Improvement (CGI) ${ }^{63,64}$ scale as 1 (very much improved) or 2 (much improved), and the mean change from baseline at $\mathrm{t} 1$ and $\mathrm{t} 2 \mathrm{on}$ the Liebowitz Social Anxiety Scale ${ }^{65-67}$ (LSAS) total score. The LSAS is a 24-item assessment of fear and avoidance of several public-social situations. Statistical analysis of the obtained data was performed using $t$-test, and $P<0.05$ was considered statistically significant.

\section{Radio electric asymmetric conveyer}

The REAC ${ }^{68,69}$ is a medical device that is based on an innovative technology for biostimulation and/or bioenhancement techniques. The model used in this study (Convogliatore di Radianza Modulante, ASMED, Florence, Italy) is specific for noninvasive brain stimulation techniques.

The NPPO-REAC treatment protocol consisted of seven radiofrequency bursts of $500 \mathrm{~ms}$ each at a frequency of 10.5 $\mathrm{GHz}$ and a specific absorption rate of $7 \mu \mathrm{W} / \mathrm{kg}$, applied by touching the metallic tip of the REAC probe to the ear pavilion.

\section{Results}

Both treatments were well tolerated, and no patients suspended the study because of any side effects. At baseline, in both the NPPO-REAC and sertraline groups, for fear and avoidance, a marked clinical picture was detected (Figures 1 and 2).

Ten $(43.5 \%)$ of 23 subjects on NPPO-REAC and six $(30.0 \%)$ of the 20 taking sertraline were much improved or

Table I Demographics

\begin{tabular}{lllll}
\hline Treatment & Patients & Male & Female & Mean age (years) \\
\hline NPPO-REAC & 23 & 8 & 15 & $31.4 \pm 2.3$ \\
Sertraline & 20 & 5 & 15 & $30.7 \pm 2.8$ \\
\hline
\end{tabular}

Abbreviations: NPPO, neuro psycho physical optimization; REAC, radio electric asymmetric conveyor. 


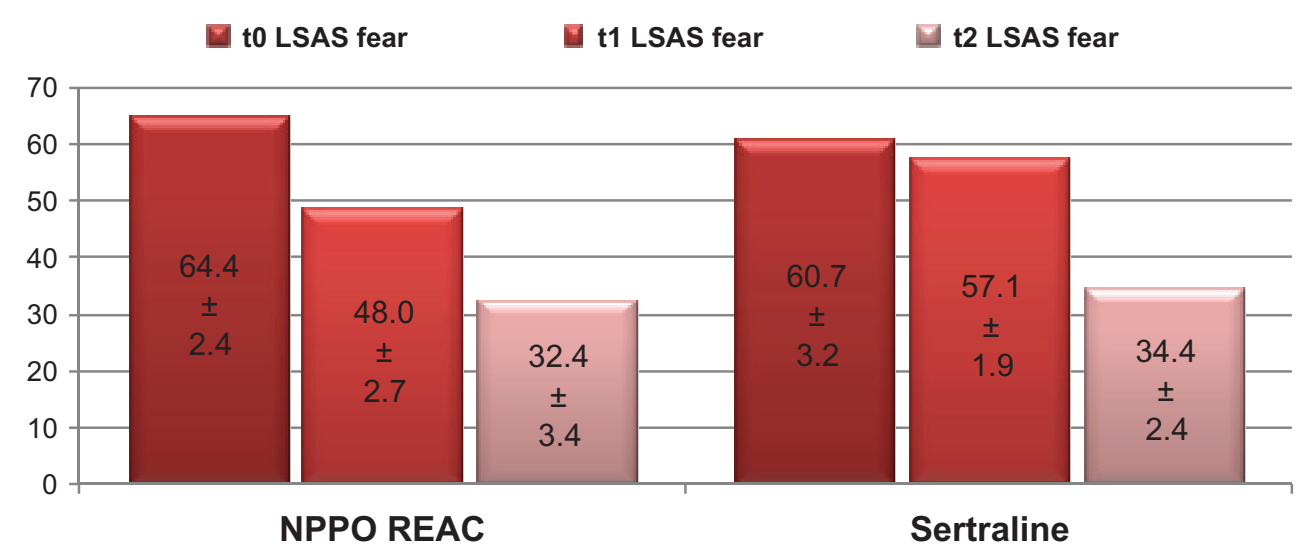

Figure I Liebowitz Social Anxiety Scale (LSAS) score decreasing for fear.

very much improved 1 month after the first NPPO-REAC cycle (t1) (Table 2). Sixteen (69.6\%) of the subjects on NPPOREAC and ten $(50.0 \%)$ of the subjects taking sertraline were much improved or very much improved 1 month after the second NPPO-REAC cycle (t2) (Table 2). The proportion of NPPO-REAC responders (ie, a CGI score of 1 or 2) was significantly greater than for sertraline (Table 2).

At $\mathrm{t} 1$, for NPPO-REAC, LSAS total fear score decreased from $64.4 \pm 2.4$ to $48.0 \pm 2.7$ (Figure 1) and for sertraline from $60.7 \pm 3.2$ to $57.1 \pm 1.9$ ( $t$-test $t=-12.595, \mathrm{DF}=41$, $P=0.000$ ) (Figure 1); for NPPO-REAC, LSAS total avoidance score decreased from $67.2 \pm 4.3$ to $50.0 \pm 3.2$ and for sertraline from $62.4 \pm 2.7$ to $54.2 \pm 2.3$ ( $t$-test $t=-4.873$, $\mathrm{DF}=41, P=0.000)$ (Figure 2).

From baseline to t1, gSAD improved from marked to moderate both for fear (Figure 1) and for avoidance (Figure 2) in the NPPO-REAC treatment group, and only for avoidance in the sertraline group.

At $\mathrm{t} 2$, for NPPO-REAC, LSAS total fear score decreased from $48.0 \pm 2.7$ to $32.4 \pm 3.4$ (Figure 1) and for sertraline from
$57.1 \pm 1.9$ to $34.4 \pm 2.4(t$-test $t=-2.196, \mathrm{DF}=41, P<0.05)$ (Figure 1); for NPPO-REAC, LSAS total avoidance score decreased from $50.0 \pm 3.2$ to $30.5 \pm 2.9$ (Figure 2) and for sertraline from $54.2 \pm 2.3$ to $32.0 \pm 2.2$ ( $t$-test $t=-1.888$, $\mathrm{DF}=41, P=\mathrm{NS})$ (Figure 2).

From baseline to 2 , in both the NPPO-REAC and the sertraline groups, for fear (Figure 1) and avoidance (Figure 2), gSAD improved from marked to subthreshold for both fear and avoidance for NPPO-REAC.

\section{Discussion and conclusion}

This is probably the first study that compares the efficacy of a brain stimulation technique with a targeted drug, sertraline, in the treatment of gSAD. The results clearly demonstrate that NPPO-REAC treatment effectively and quickly reduces the core symptoms and the avoidance associated with this disorder. In this research, NPPO-REAC was globally statistically superior to standard dosages of sertraline on selected primary efficacy criteria, CGI improvement, and LSAS total score for fear and avoidance. Considering the early age at onset

to LSAS avoidance

t1 LSAS avoidance 2

t2 LSAS avoidance 2

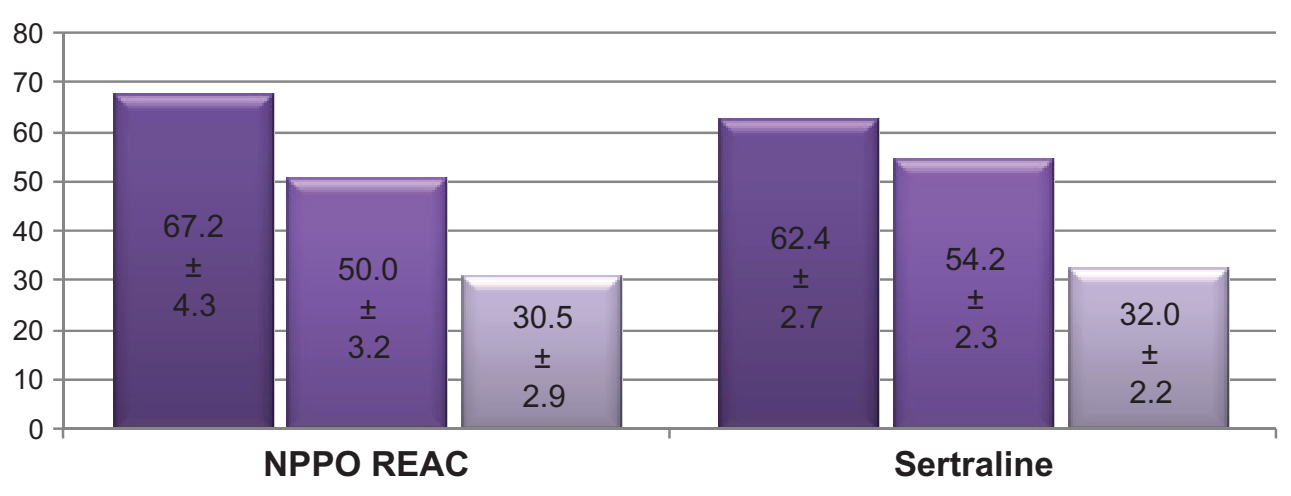

Figure 2 Liebowitz Social Anxiety Scale (LSAS) score decreasing for avoidance. 
Table 2 Clinical Global Improvement (CGI)

\begin{tabular}{|c|c|c|c|c|}
\hline \multirow[t]{2}{*}{ CGI } & \multicolumn{4}{|l|}{$\begin{array}{l}\text { Number } \\
\text { of patients }\end{array}$} \\
\hline & $\begin{array}{l}\text { REAC } \\
\mathrm{tl}\end{array}$ & $\begin{array}{l}\text { REAC } \\
\text { t2 }\end{array}$ & $\begin{array}{l}\text { Sertraline } \\
\text { tl }\end{array}$ & $\begin{array}{l}\text { Sertraline } \\
\text { t2 }\end{array}$ \\
\hline $\begin{array}{l}\text { Very much } \\
\text { improved or } \\
\text { much improved }\end{array}$ & $10(43.5 \%)$ & $16(69.6 \%)$ & $6(30 \%)$ & $10(50 \%)$ \\
\hline
\end{tabular}

Abbreviations: NPPO, neuro psycho physical optimization; REAC, radio electric asymmetric conveyor; $\mathrm{tl}$, first cycle; $\mathrm{t} 2$, second cycle.

and chronic course of this disorder, detecting a reduction in disability after only a 6-month follow-up is noteworthy. It is hoped, but remains to be shown in future studies, that longer duration and more cycles of NPPO-REAC treatment might result in even further structuration of results and, consequently, in the reduction of functional impairment.

As previously demonstrated in the treatment of agoraphobia, also in this study a sort of cognitive restructuration performed by NPPO-REAC has been highlighted, in order to guarantee the patient a more rational control of emotions when exposed to social and performance situations and, consequently, better management of the pattern of avoidance behaviors.

In respect of the CGI scale, the greater percentage of patients who felt themselves to be much improved or very much improved with NPPO-REAC than with sertraline highlights the deeper action of the gentle NPPO-REAC brain stimulation compared with the drug.

Another aspect of particular interest is the feeling of natural and no artificial improvement of gSAD symptomatology. This is very important, because typical of anxiety disorders, and probably one of the main reasons of the premature discontinuation of the drug treatment, is the feeling of the artificiality of the results. Therefore, from this point of view, the NPPO-REAC provides greater adherence to the treatment of these patients.

Obviously, there are a number of limitations to this study. $\mathrm{SAD}$ is a chronic and disabling disease that in any cases may require long-term therapy. Because of its design, this study did not accurately assess NPPO-REAC efficacy beyond the short, 6-month treatment period. It is possible, although not yet proven, that a longer course of therapy could result in sustained and even greater reductions in impairment and in improvement in quality of life.

Indeed, further studies of long-term treatment with NPPO-REAC are needed to determine the optimal duration of therapy, the number of NPPO-REAC cycles needed, and the efficacy in sustaining remission.
Because NPPO-REAC is also effective in the treatment of depressive symptoms, another limitation of this study is its inability to definitively demonstrate that reductions in social anxiety symptoms were not simply a secondary manifestation of this antidepressant effect.

\section{Conclusion}

NPPO-REAC is an effective treatment for SAD, allowing substantial and clinically meaningful reductions in symptoms and disability, in comparison with sertraline.

\section{Disclosure}

Salvatore Rinaldi and Vania Fontani are the inventors of the radio electric asymmetric conveyor.

\section{References}

1. Weeks JW, Heimberg RG, Rodebaugh TL, et al. Psychometric evaluation of the fear of positive evaluation scale in patients with social anxiety disorder. Psychol Assess. October 3, 2011.

2. Schneier FR, Rodebaugh TL, Blanco C, et al. Fear and avoidance of eye contact in social anxiety disorder. Compr Psychiatry. 2011; 52(1):81-87.

3. Zimmerman M, Dalrymple K, Chelminski I, et al. Recognition of irrationality of fear and the diagnosis of social anxiety disorder and specific phobia in adults: implications for criteria revision in DSM-5. Depress Anxiety. 2010;27(11):1044-1049.

4. Moitra E, Herbert JD, Forman EM. Behavioral avoidance mediates the relationship between anxiety and depressive symptoms among social anxiety disorder patients. J Anxiety Disord. 2008;22(7): $1205-1213$.

5. Hofmann SG, Bitran S. Sensory-processing sensitivity in social anxiety disorder: relationship to harm avoidance and diagnostic subtypes. J Anxiety Disord. 2007;21(7):944-954.

6. Kashdan TB, Morina N, Priebe S. Post-traumatic stress disorder, social anxiety disorder, and depression in survivors of the Kosovo War: experiential avoidance as a contributor to distress and quality of life. J Anxiety Disord. 2009;23(2):185-196.

7. Barrera TL, Norton PJ. Quality of life impairment in generalized anxiety disorder, social phobia, and panic disorder. J Anxiety Disord. 2009;23(8):1086-1090.

8. Hambrick JP, Turk CL, Heimberg RG, et al. The experience of disability and quality of life in social anxiety disorder. Depress Anxiety. 2003;18(1):46-50.

9. Masia-Warner C, Klein RG, Dent HC, et al. School-based intervention for adolescents with social anxiety disorder: results of a controlled study. J Abnorm Child Psychol. 2005;33(6):707-722.

10. Fisher PH, Masia-Warner C, Klein RG. Skills for social and academic success: a school-based intervention for social anxiety disorder in adolescents. Clin Child Fam Psychol Rev. 2004;7(4):241-249.

11. Furukawa TA, Chen J, Watanabe N, et al. Videotaped experiments to drop safety behaviors and self-focused attention for patients with social anxiety disorder: do they change subjective and objective evaluations of anxiety and performance? J Behav Ther Exp Psychiatry. 2009;40(2):202-210.

12. Xu Y, Schneier F, Heimberg RG, et al. Gender differences in social anxiety disorder: Results from the national epidemiologic sample on alcohol and related conditions. J Anxiety Disord. August 17, 2011.

13. Bacon AK, Ham LS. Attention to social threat as a vulnerability to the development of comorbid social anxiety disorder and alcohol use disorders: an avoidance-coping cognitive model. Addict Behav. 2010;35(11):925-939. 
14. Schneier FR, Foose TE, Hasin DS, et al. Social anxiety disorder and alcohol use disorder co-morbidity in the National Epidemiologic Survey on Alcohol and Related Conditions. Psychol Med. 2010;40(6): 977-988.

15. Chou KL. Social anxiety disorder in older adults: evidence from the National Epidemiologic Survey on alcohol and related conditions. J Affect Disord. 2009;119(1-3):76-83.

16. Buckner JD, Turner RJ. Social anxiety disorder as a risk factor for alcohol use disorders: a prospective examination of parental and peer influences. Drug Alcohol Depend. 2009;100(1-2):128-137.

17. Akechi T, Okuyama T, Sagawa R, et al. Social anxiety disorder as a hidden psychiatric comorbidity among cancer patients. Palliat Support Care. 2011;9(1):103-105.

18. Dalrymple KL, Zimmerman M. Screening for social fears and social anxiety disorder in psychiatric outpatients. Compr Psychiatry. 2008;49(4):399-406.

19. Bruce SE, Yonkers KA, Otto MW, et al. Influence of psychiatric comorbidity on recovery and recurrence in generalized anxiety disorder, social phobia, and panic disorder: a 12-year prospective study. Am J Psychiatry. 2005;162(6):1179-1187.

20. Lin CC. Duloxetine treatment of social anxiety disorder with comorbid major depression. J Clin Psychopharmacol. 2008;28(5):591-592; author reply $592-593$.

21. Schneier FR, Blanco C, Campeas R, et al. Citalopram treatment of social anxiety disorder with comorbid major depression. Depress Anxiety. 2003;17(4):191-196.

22. Bronisch T, Hecht H. Major depression with and without a coexisting anxiety disorder: social dysfunction, social integration, and personality features. J Affect Disord. 1990;20(3):151-157.

23. Moitra E, Beard C, Weisberg RB, Keller MB. Occupational impairment and social anxiety disorder in a sample of primary care patients. JAffect Disord. 2011;130(1-2):209-212.

24. Culpepper L. Social anxiety disorder in the primary care setting. J Clin Psychiatry. 2006;67 Suppl 12:31-37.

25. Kashdan TB, Frueh BC, Knapp RG, et al. Social anxiety disorder in veterans affairs primary care clinics. Behav Res Ther. 2006; 44(2):233-247.

26. Gross R, Olfson M, Gameroff MJ, et al. Social anxiety disorder in primary care. Gen Hosp Psychiatry. 2005;27(3):161-168.

27. Elliott HW, Reifler B. Social anxiety disorder. A guide for primary care physicians. NC Med J. 2000;61(3):176-178.

28. Katzelnick DJ, Greist JH. Social anxiety disorder: an unrecognized problem in primary care. J Clin Psychiatry. 2001;62 Suppl 1:11-15; discussion $15-16$.

29. Sheeran T, Zimmerman M. Social phobia: still a neglected anxiety disorder? J Nerv Ment Dis. 2002;190(11):786-788.

30. Liebowitz MR, Gorman JM, Fyer AJ, Klein DF. Social phobia. Review of a neglected anxiety disorder. Arch Gen Psychiatry. 1985 42(7):729-736.

31. Fink M, Akimova E, Spindelegger C, et al. Social anxiety disorder: epidemiology, biology and treatment. Psychiatr Danub. 2009; 21(4):533-542.

32. Khalid-Khan S, Santibanez MP, McMicken C, Rynn MA. Social anxiety disorder in children and adolescents: epidemiology, diagnosis, and treatment. Paediatr Drugs. 2007;9(4):227-237.

33. Grant BF, Hasin DS, Blanco C, et al. The epidemiology of social anxiety disorder in the United States: results from the National Epidemiologic Survey on Alcohol and Related Conditions. J Clin Psychiatry. 2005;66(11):1351-1361.

34. Ha J, Lim SW, Shin YC, Oh KS. Comparison of anxiety-related traits between generalized and nongeneralized subtypes of social anxiety disorder. J Nerv Ment Dis. 2011;199(6):390-393.

35. Cox BJ, Turnbull DL, Robinson JA, et al. The effect of avoidant personality disorder on the persistence of generalized social anxiety disorder in the general population: results from a longitudinal, nationally representative mental health survey. Depress Anxiety. 2011;28(3): 250-255.
36. Moscovitch DA, Suvak MK, Hofmann SG. Emotional response patterns during social threat in individuals with generalized social anxiety disorder and non-anxious controls. J Anxiety Disord. 2010;24(7):785-791.

37. Shah SG, Klumpp H, Angstadt M, et al. Amygdala and insula response to emotional images in patients with generalized social anxiety disorder. J Psychiatry Neurosci. 2009;34(4):296-302.

38. Blanco C, Antia SX, Liebowitz MR. Pharmacotherapy of social anxiety disorder. Biological Psychiatry. 2002;51(1):109-120.

39. Warwick JM, Carey P, Van der Linden G, et al. A comparison of the effects of citalopram and moclobemide on resting brain perfusion in social anxiety disorder. Metab Brain Dis. 2006;21(2-3):241-252.

40. Stein DJ, Cameron A, Amrein R, Montgomery SA. Moclobemide is effective and well tolerated in the long-term pharmacotherapy of social anxiety disorder with or without comorbid anxiety disorder. Int Clin Psychopharmacol. 2002;17(4):161-170.

41. Stein MB, Seedat S, Gelernter J. Serotonin transporter gene promoter polymorphism predicts SSRI response in generalized social anxiety disorder. Psychopharmacology (Berl). 2006;187(1):68-72.

42. Vasile RG, Bruce SE, Goisman RM, et al. Results of a naturalistic longitudinal study of benzodiazepine and SSRI use in the treatment of generalized anxiety disorder and social phobia. Depress Anxiety. 2005;22(2):59-67.

43. Van Ameringen M, Mancini C, Farvolden P, Oakman J. Drugs in development for social anxiety disorder: more to social anxiety than meets the SSRI. Expert Opin Investig Drugs. 2000;9(10):2215-2231.

44. Stangier U, Schramm E, Heidenreich T, et al. Cognitive therapy vs interpersonal psychotherapy in social anxiety disorder: a randomized controlled trial. Arch Gen Psychiatry. 2011;68(7):692-700.

45. Stein DJ, Ipser JC. Combined pharmacotherapy and psychotherapy for social anxiety disorder. Curr Psychiatry Rep. 2010;12(4):273-275.

46. Holmberg N, Kahkonen S. Cognitive psychotherapy of social phobia and generalized anxiety disorder. Duodecim. 2009;125(18):1949-1956.

47. Lipsitz JD, Marshall RD. Alternative psychotherapy approaches for social anxiety disorder. Psychiatr Clin North Am. 2001;24(4):817-829.

48. Castagna A, Rinaldi S, Fontani V, et al. Does osteoarthritis of the knee also have a psychogenic component? Psycho-emotional treatment with a radio-electric device vs intra-articular injection of sodium hyaluronate: an open-label, naturalistic study. Acupunct Electrother Res. 2010;35(1-2):1-16.

49. Collodel G, Moretti E, Fontani V, et al. Effect of emotional stress on sperm quality. Indian J Med Res. 2008;128(3):254-261.

50. Rinaldi S, Fontani V, Aravagli L, Mannu P. Psychometric evaluation of a radio electric auricular treatment for stress related disorders: a doubleblinded, placebo-controlled controlled pilot study. Health Qual Life Outcomes. 2010;8:31.

51. Rinaldi S, Fontani V, Aravagli L, et al. Stress-related psycho-physiological disorders: randomized single blind placebo controlled naturalistic study of psychometric evaluation using a radio electric asymmetric treatment. Health Qual Life Outcomes. 2011;9(1):54.

52. Rinaldi S, Fontani V, Aravagli L, Margotti ML. Psychological and symptomatic stress-related disorders with radio-electric treatment: psychometric evaluation. Stress and Health. 2010;26(5):350-358.

53. Rinaldi S, Fontani V, Moretti E, et al. A new approach on stressrelated depression and anxiety: neuro-psycho-physical-optimization with radio electric asymmetric conveyer. Indian J Med Res. 2010;132: 189-194.

54. Olivieri EB, Vecchiato C, Ignaccolo N, et al. Radioelectric brain stimulation in the treatment of generalized anxiety disorder with comorbid major depression in a psychiatric hospital: a pilot study. Neuropsychiatr Dis Treat. 2011;7:449-455.

55. Mannu P, Rinaldi S, Fontani V, et al. Radio electric treatment vs Es-citalopram in the treatment of panic disorders associated with major depression: an open-label, naturalistic study. Acupunct Electrother Res. 2009;34(3-4):135-149.

56. Mannu P, Rinaldi S, Fontani V, Castagna A. Long-term treatment of bipolar disorder with a radioelectric asymmetric conveyor. Neuropsychiatr Dis Treat. 2011;7:373-379. 
57. Mannu P, Rinaldi S, Fontani V, Castagna A. Radio electric asymmetric brain stimulation in the treatment of behavioral and psychiatric symptoms in Alzheimer disease. Clin Interv Aging. 2011;6:207-211.

58. Germans S, Van Heck GL, Masthoff ED, et al. Diagnostic efficiency among psychiatric outpatients of a self-report version of a subset of screen items of the Structured Clinical Interview for DSM-IV-TR Personality Disorders (SCID-II). Psychol Assess. 2010;22(4):945-952.

59. Hajebi A, Motevalian A, Amin-Esmaeili M, et al. Telephone versus face-to-face administration of the Structured Clinical Interview for Diagnostic and Statistical Manual of Mental Disorders, Fourth Edition, for diagnosis of psychotic disorders. Compr Psychiatry. August 5, 2011.

60. Connor KM, Davidson JR, Chung H, et al. Multidimensional effects of sertraline in social anxiety disorder. Depress Anxiety. 2006;23(1):6-10.

61. Sokolenko M, Kutcher S. Sertraline for social anxiety disorder. Expert Rev Neurother. 2003;3(6):787-795.

62. Liebowitz MR, DeMartinis NA, Weihs K, et al. Efficacy of sertraline in severe generalized social anxiety disorder: results of a double-blind, placebo-controlled study. J Clin Psychiatry. 2003;64(7):785-792.
63. Busner J, Targum SD, Miller DS. The Clinical Global Impressions scale: errors in understanding and use. Compr Psychiatry. 2009;50(3):257-262.

64. Busner J, Targum SD. The clinical global impressions scale: applying a research tool in clinical practice. Psychiatry (Edgmont). 2007;4(7):28-37.

65. Rytwinski NK, Fresco DM, Heimberg RG, et al. Screening for social anxiety disorder with the self-report version of the Liebowitz Social Anxiety Scale. Depress Anxiety. 2009;26(1):34-38.

66. Kummer A, Cardoso F, Teixeira AL. Frequency of social phobia and psychometric properties of the Liebowitz social anxiety scale in Parkinson's disease. Mov Disord. 2008;23(12):1739-1743.

67. Mennin DS, Fresco DM, Heimberg RG, et al. Screening for social anxiety disorder in the clinical setting: using the Liebowitz Social Anxiety Scale. J Anxiety Disord. 2002;16(6):661-673.

68. Rinaldi S, Fontani V. Radioelectric asymmetric conveyer for therapeutic use. US patent EP1301241 (B1). October 11, 2006, 2000.

69. Rinaldi S, Fontani V. Radioelectric asymmetric conveyer for therapeutic use. US patent 7,333,859. February 19, 2008, 2001.
Patient Preference and Adherence

\section{Publish your work in this journal}

Patient Preference and Adherence is an international, peer-reviewed, open access journal focusing on the growing importance of patient preference and adherence throughout the therapeutic continuum. Patient satisfaction, acceptability, quality of life, compliance, persistence and their role in developing new therapeutic modalities and compounds to

\section{Dovepress}

optimize clinical outcomes for existing disease states are major areas of interest. This journal has been accepted for indexing on PubMed Central. The manuscript management system is completely online and includes a very quick and fair peer-review system. Visit http://www.dovepress.com/ testimonials.php to read real quotes from published authors. 